\title{
Rossby waves with linear topography in barotropic fluids*
}

\author{
YANG Liangui (杨联贵 $)^{\dagger}$, DA Chaojiu (达朝究 $)^{\dagger,+\dagger}$ SONG Jian $(\text { 宋建 })^{\dagger,+\dagger \dagger}$, \\ ZHANG Huiqin (张会琴) ${ }^{\dagger \dagger \dagger}$, YANG Hongli (杨红丽) ${ }^{\dagger+\dagger \dagger \dagger}$, \\ HOU Yijun (侯一笏) \\ ${ }^{\dagger}$ School of Mathematical Sciences, Inner Mongolia University, Hohhot 010021, China \\ ${ }^{+t}$ College of Computer Sciences and Information Engineering, Northwest University for Nationalities, Lanzhou 730124, China

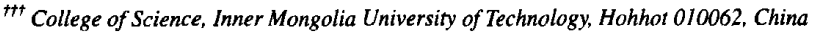 \\ ${ }^{t+t t}$ Institute of Mechanics, Chinese Academy of Sciences, Beijing 100080, China \\ ${ }^{\dagger t t+t}$ Institute of Oceanology, Chinese Academy of Sciences, Qingdao 266071, China
}

Received Nov. 13, 2006; revision accepted Apr. 27, 2007

\begin{abstract}
Rossby waves are the most important waves in the atmosphere and ocean, and are parts of a large-scale system in fluid. The theory and observation show that, they satisfy quasi-geostrophic and quasi-static equilibrium approximations. In this paper, solitary Rossby waves induced by linear topography in barotropic fluids with a shear flow are studied. In order to simplify the problem, the topography is taken as a linear function of latitude variable $y$, then employing a weakly nonlinear method and a perturbation method, a KdV (Korteweg-de Vries) equation describing evolution of the amplitude of solita度数你店y waves induced by linear topography is derived. The results show that the variation of linear topography can induce the solitary Rossby waves in barotropic fluids with a shear flow, and extend the classical geophysical theory of fluid dynamics.
\end{abstract}

Keyword: nonlinear Rossby waves; KdV equation; topography effect; perturbation method

\section{INTRODUCTION}

Rossby waves, satisfying the quasi-geostrophic and quasi-static equilibrium approximations, are the most important large-scale waves in the atmosphere and ocean. Long (1964) and Benney (1966) discussed long waves in barotropic fluid in homogeneous atmosphere and obtained a $\mathrm{KdV}$ equation. However, these previous studied were limited to the case where velocity shear was small in basic uniform zonal motion and could not apply to stream-line-flow case with waves. The limitation superimposed on an order-one uniform flow and ignored special considerations on the existence of a critical layer where wave speed matches zonal-flow velocity. On the other hand, solitary Rossby waves were studied by Larsen (1965) and Clarke (1971), but again, they did not discuss the critical layer nor provide any information about possible flow patterns. Redekopp (1977) and Wadati (1973) discussed the general theory of solitary waves in zonal, planetary shear flow, focused on two special atmospheric model and demonstrated that the amplitude of long Rossby wave propagating in a zonal shear flow was governed by Korteweg-de Vries $(\mathrm{KdV})$ equation or modified Korteweg-de Vries (mKdV) equation depending on the distribution of the atmospheric density stratification. Redekopp and Weidman (1978) discussed the solitary Rossby waves in a zonal shear flow and their interactions. A coupled nonlinear evolution $\mathrm{KdV}$ equation was derived for the interaction among solitary waves of different long-wave phase velocities propagating in a zonal shear flow. Maslowe and Redekop (1980) discussed long nonlinear waves in stratified flows and analyzed the effect of shear on long waves in a stratified flow. However, they did not discuss the topography effect on the Rossby waves. Boyd (1980) applied multiple scales to the original equation and showed that long

\footnotetext{
* Supported by the Knowledge Innovation Program of Chinese Academy of Sciences (KZCX1-YW-12), Scientific Research Foundation for the Returned Overseas Chinese Scholar, and by Natural Science Foundation of Inner Mongolia (200408020112)

** Corresponding author: yjhou@ms.qdio.ac.cn
} 
and small Rossby waves were evolved in longitude and time shown by nonlinear $\mathrm{KdV}$ equation or mKdV equation. Liu and Tan (1992) studied and discussed Rossby waves with Rossby parameter $\beta$ with latitude and extend the $\beta$-plane approximation into $f=f_{0}+\beta_{0} y-(1 / 2) \gamma_{0} y^{2}$. Luo $(1991,1995)$ discussed the solitary Rossby waves with the same parameter and dipole blocking using extended $\beta$-plane approximation. Zhao (1997) examined the dynamical influence of topography on ultra-long Rossby waves in long-latitude atmosphere, and concluded that the topographical forcing could lead to instability of ultra-long Rossby waves. Zhao et al. (2001) observed equatorial envelope solitary Rossby waves in shear flow, applied a simple shallow-water model on an equatorial $\beta$-plane with asymptotic method of multiple scales to nonlinear equatorial solitary Rossby waves in mean zonal flow with meridional shear, and yielded a cubic nonlinear Schrödinger equation, satisfying large amplitude equatorial envelope solitary Rossby waves in shear flow, after studying the effect of basic flow shear on the nonlinear solitary Rossby waves. In addition,

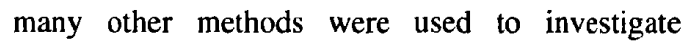
large-scale waves (Su et al., 2006; Li et al., 2006; Sun et al., 2006).

In this paper, solitary Rossby waves induced by linear topography are studied. To simplify the problem, the topography was taken as a linear function of latitude variable $y$. The results show that the variation of linear topography can induce the solitary Rossby waves in barotropic fluids.

\section{DERIVATION OF THE KDV EQUATION}

\subsection{Governing equation and boundary conditions}

The basic equation of motion used in this study is the quasi-geostrophic equation in form of potential-vorticity (Zhu et al., 1991; Wang and Weng, 1981) for shallow water with the assumption that $\beta$ effect can be neglected and the topography is a linear function of latitude variable $y$.

$$
\left(\frac{\partial}{\partial t}+\frac{\partial}{\partial x} \psi \frac{\partial}{\partial y}-\frac{\partial}{\partial y} \psi \frac{\partial}{\partial x}\right)\left(\nabla^{2} \psi+\frac{f_{0}}{H} h_{b} y\right)=0
$$

where $H$ is the average depth of the flow, $f_{0}$ is the local Corilolis parameter, and $h_{b}$ is the slope of the topography in latitudinal $(y)$ directions, and all of them could be constants in specific cases; $y=\mathrm{a}\left(\varphi-\varphi_{0}\right)$ is latitude variable; $\nabla^{2}$ is the Laplace operator.
The rigid-wall side boundary conditions are defined as:

$$
\psi\left(y_{1}\right)=\psi\left(y_{2}\right)=0
$$

where $y=y_{1}, y=y_{2}$ denote the southern and northern edges of the zonal flow in which latitudinal boundaries may exist. It is convenient to convert (1) into non-dimensional form by taking the following scaling rules:

$$
\begin{aligned}
(x, y) & =L_{0}\left(x^{*}, y^{*}\right), \quad t=\frac{L_{0}}{U_{0}} t^{*}, \psi=U_{0} L_{0} \psi^{*}, \\
h_{b} & =\frac{H}{f_{0}} \frac{U_{0}}{L_{0}^{2}} h_{b}^{*}
\end{aligned}
$$

where non-dimensional variables are marked by an asterisk. $L_{0}$ is the characteristic measure of length of the mean zonal flow, and $U_{0}$ is the characteristic velocity scale. Substitution of (3) into (1) yields:

$$
\begin{aligned}
& \left(\frac{\partial}{\partial t^{*}}+\frac{\partial}{\partial x^{*}} \psi^{*} \frac{\partial}{\partial y^{*}}-\frac{\partial}{\partial y^{*}} \psi^{*} \frac{\partial}{\partial x^{*}}\right) \\
& \left(\nabla^{2} \psi^{*}+h_{b}^{*} y^{*}\right)=0
\end{aligned}
$$

where the asterisk can be dropped for simplicity, and can be rewritten as:

$$
\left(\frac{\partial}{\partial t}+\frac{\partial}{\partial x} \psi \frac{\partial}{\partial y}-\frac{\partial}{\partial y} \psi \frac{\partial}{\partial x}\right)\left(\nabla^{2} \psi+h_{b} y\right)=0
$$

and the boundary conditions (2) can be rewritten in the form of:

$$
\psi(0)=\psi(1)=0
$$

\subsection{Derivation of the $K d V$ equation}

We assume that the basic stream function has a form of:

$$
\Psi(y)=-\int\left[U(y)-c_{0}\right] d y
$$

where $c_{0}$ is a constant, an eigenvalue of the eigenvalue problem below. The Eq.7 means actually that we have taken a traveling wave transformation:

$$
x=x-c_{0} t
$$

It is clear that the shear flow is $U(y)$. We take total stream function $\psi$ as the disturbance stream $\psi$ in a non-dimensional amplitude $\varepsilon$ with 
superimposition of zonal flow $\psi(y)$. When $\varepsilon<<1$, it is a weakly nonlinear problem, with which this paper deals. The total stream function thus becomes:

$$
\psi=\Psi(y)+\varepsilon \psi^{\prime}=-\int\left[U(y)-c_{0}\right] d y+\varepsilon \psi^{\prime}
$$

Hereafter, all the expressions are written in non-dimensional form and all symbols stand for dimensional quantities. Substituting (9) with (5), and neglecting the apostrophe of the disturbance stream function for simplicity, yield:

$$
\begin{aligned}
& \varepsilon \frac{\partial}{\partial t} \nabla^{2} \psi+\varepsilon\left(h_{b}-U^{\prime \prime}\right) \frac{\partial \psi}{\partial x}+ \\
& \varepsilon\left(U-c_{0}\right) \frac{\partial}{\partial x} \nabla^{2} \psi+\varepsilon^{2} J\left[\psi, \nabla^{2} \psi\right]=0
\end{aligned}
$$

where $U^{\prime \prime}$ is the second derivative of $U$ and $J[A, B]=(\partial A / \partial x)(\partial B / \partial y)-(\partial A / \partial y)(\partial B / \partial x) \quad$ is the Jacobi operator.

Simplifying (10) into:

$$
\begin{aligned}
& \frac{\partial}{\partial t} \nabla^{2} \psi+\left(h_{b}-U^{\prime \prime}\right) \frac{\partial \psi}{\partial x}+ \\
& \text { 万方数据 } \\
& \quad\left(U-c_{0}\right) \frac{\partial}{\partial x} \nabla^{2} \psi+\varepsilon J\left[\psi, \nabla^{2} \psi\right]=0
\end{aligned}
$$

where $\varepsilon$ is a measure of the magnitude of nonlinear products. Here we focus on the systems in which nonlinearity and dispersion are in the same order of magnitude.

The asymptotic solution to the weakly nonlinear problem with multiple scale method is studied below. Slow time $T$ and long space $X$ scales are incorporated with (11) by derivative transformations:

$$
\frac{\partial}{\partial x}=\varepsilon^{1 / 2} \frac{\partial}{\partial X}, \frac{\partial}{\partial t}=\varepsilon^{3 / 2} \frac{\partial}{\partial T}, \frac{\partial^{2}}{\partial x^{2}}=\varepsilon \frac{\partial^{2}}{\partial X^{2}}
$$

where $T$ and $X$ are defined as:

$$
X=\varepsilon^{1 / 2} x, T=\varepsilon^{3 / 2} t
$$

Substituting (12) and (13) into (11), yields:

$$
\varepsilon^{\frac{1}{2}} L_{0}(\psi)+\varepsilon^{\frac{3}{2}} L_{1}(\psi)+\varepsilon^{\frac{3}{2}} J\left[\psi, \frac{\partial^{2} \psi}{\partial y^{2}}\right]+O\left(\varepsilon^{\frac{5}{2}}\right)=0
$$

Only variables $X$ and $T$ are included in Eq. 14, variables $x$ and $t$ are eliminated; $J[A, B]=$ $(\partial A / \partial X)(\partial B / \partial y)-(\partial A / \partial y)(\partial B / \partial X)$. It is convenient to introduce two linear differential operators defined as:

$$
\begin{aligned}
& L_{0}=\left[\left(U-c_{0}\right) \frac{\partial^{2}}{\partial y^{2}}+\left(h_{b}-U^{\prime \prime}\right)\right] \frac{\partial}{\partial X} \\
& L_{1}=\frac{\partial}{\partial T} \frac{\partial^{2}}{\partial y^{2}}+\left(U-c_{0}\right) \frac{\partial^{3}}{\partial X^{3}}
\end{aligned}
$$

Supposing that the disturbance stream function has the form of (Jeffrey and Kawahara, 1982)

$$
\psi=\psi^{(1)}+\varepsilon \psi^{(2)}+\varepsilon^{2} \psi^{(3)}+\cdots
$$

Substitution of (17) into (14) and (6) yields the first order:

$$
\begin{aligned}
& L_{0}\left[\psi^{(1)}\right]=0 \\
& \psi^{(1)}(0)=\psi^{(1)}(1)=0
\end{aligned}
$$

Eq.18 is a linear differential equation. Assuming that $\psi^{(1)}$ has the form of:

$$
\psi^{(1)}=A(X, T) \Phi^{(1)}(y)
$$

Substitution of (20) into (18) and (19) yields

$$
\begin{aligned}
& \left(\frac{d^{2}}{d y^{2}}+\frac{h_{b}-U^{\prime \prime}}{U-c_{0}}\right) \Phi^{(1)}=0 \\
& \Phi^{(1)}(0)=\Phi^{(1)}(1)=0
\end{aligned}
$$

In Eq.21, $U-c_{0} \neq 0$ is assumed. Eqs. 21 and 22 define an eigenvalue problem for the eigenvalue $c_{0}$. Once $h_{b}$ and $U(y)$ are specified, $\Phi^{(1)} y$ can be determined. In other words, the space structure of the wave along $y$-direction can be described once the distributions of linear topography and $U(y)$ are given. Since $U(y)$ is a nonlinear function of variable $y$, it is difficult to get the analytic solution of this eigenvalue problem. In addition, clearly, $O\left(\varepsilon^{1 / 2}\right)$ is a time-invariant system. However, the evolution of 
the amplitude of the solitary Rossby waves could not be determined. In order to derive the evolution of the amplitude $A(X, T)$ of the wave, solutions of the higher order problem are sought, such as $O\left(\varepsilon^{3 / 2}\right)$ :

$$
L_{0}\left[\psi^{(2)}\right]=-\left\{L_{1}\left[\psi^{(1)}\right]+J\left[\psi^{(1)}, \frac{\partial^{2}}{\partial y^{2}} \psi^{(1)}\right]\right\} \equiv F_{2}
$$

$$
\psi^{(2)}(0)=\psi^{(2)}(1)=0
$$

where $F_{2}=-\left\{L_{1}\left[\psi^{(1)}\right]+J\left[\psi^{(1)}, \partial^{2} \psi^{(1)} / \partial y^{2}\right]\right\}$. It is clear that there are dispersion effect in $y$-direction and weak nonlinear effect in this order. However, the effects do not exist in $O\left(\varepsilon^{1 / 2}\right)$. We take $\psi^{(2)}$ in $\psi^{(2)}$ $=B(X, T) \Phi^{(2)}(y)$, multiply both sides of Eq.(23) by $\Phi^{(1)}(y)$, and then integrate it over $[0,1]$; at the same time, by employing the identity:

$$
\begin{gathered}
\Phi^{(1)} \frac{\partial^{2}}{\partial y^{2}} \Phi^{(2)} \equiv \frac{\partial}{\partial y}\left[\Phi^{(1)} \frac{\partial}{\partial y} \Phi^{(1)}\right]- \\
\text { 万方数据(2) } \frac{\partial}{\partial y}\left[\Phi^{(1)}\right]+\Phi^{(2)} \frac{\partial^{2}}{\partial y^{2}} \Phi^{(1)}
\end{gathered}
$$

We get

$$
\int_{0}^{(1)}(y) \frac{F_{2}}{U-c_{0}} d y=0
$$

This shows, if a perturbation problem (17) has an efficient solution, the secular term $F_{2}$ must satisfy Eq.25; otherwise the amplitude of wave would be infinite and meaningless. Substitution of $F_{2}=-\left\{L_{1}\left[\psi^{(1)}\right]+J\left[\psi^{(1)}, \partial^{2} \psi^{(1)} / \partial y^{2}\right]\right\}$ and (20) into (25) yields:

$$
\begin{aligned}
& \int_{U}^{l}\left[\Phi^{(1)}(y)\right]^{2} \frac{h_{b}-U^{\prime \prime}}{\left(U-c_{0}\right)^{2}} d y \frac{\partial A}{\partial T}+ \\
& \int_{0}^{1} \frac{\left[\Phi^{(1)}(y)\right]^{3}}{U-c_{0}}\left(\frac{h_{b}-U^{\prime \prime}}{U-c_{0}}\right)_{y} d y A \frac{\partial A}{\partial X}- \\
& \int_{0}^{1}\left[\Phi^{(1)}(y)\right]^{2} d y \frac{\partial^{3} A}{\partial X^{3}}=0
\end{aligned}
$$

where $\left[\left(h_{b}-U^{\prime \prime}\right) /\left(U-c_{0}\right)\right]_{y}$ is the derivative of $\left(h_{b}-\right.$ $\left.U^{\prime \prime}\right) /\left(U-c_{0}\right)$ with respect to $y$. To simplify Eq. 26 , coefficients $I_{0}, S_{0}$ and $R_{00}$ were introduced in the definitions of:

$$
I_{0}=\int_{0}^{l}\left[\Phi^{(1)}(y)\right]^{2} \frac{h_{b}-U^{\prime \prime}}{\left(U-c_{0}\right)^{2}} d y
$$

$$
S_{0}=\frac{-\int_{0}^{1}\left[\Phi^{(1)}(y)\right]^{2} d y}{I_{0}}
$$

$$
R_{00}=\frac{\int \frac{\left[\Phi^{(1)}(y)\right]^{3}}{U-c_{0}}\left(\frac{h_{b}-U^{\prime \prime}}{U-c_{0}}\right)_{y} d y}{I_{0}}
$$

where $\Phi^{(1)}(y)$ is determined by the solution of the eigenvalue problem (21) and (22). Thus Eq. 26 has the form

$$
\frac{\partial A}{\partial T}+R_{00} A \frac{\partial A}{\partial X}+S_{0} \frac{\partial^{3} A}{\partial X^{3}}=0
$$

where the amplitude $A(X, T)$ of solitary Rossby waves satisfies the $\mathrm{KdV}$ equation. Obviously, coefficients $S_{0}$ and $R_{00}$ are related to functions $h_{b} y$ and $U(y)$. If $U(y)$ is a constant, i.e., the basic flow is not a shear flow, Eq. 30 becomes a linear KdV equation, and there is no solitary Rossby wave solution (Guo and Pang, 1987). In other words, no solitary Rossby waves occur if $U=$ constant and topography varies with latitude linearly. Therefore, nonlinear variation of topography with latitude is a necessary condition for the formation of solitary Rossby waves when $U=$ constant.

\section{CONCLUSION}

An asymptotic method and weakly nonlinear method could be used to investigate nonlinear Rossby waves in zonal shear flow in middle or high latitude zones by employing a simple shallow-water model. A nonlinear $\mathrm{KdV}$ equation, which describes the amplitude evolution and other main characteristics of solitary Rossby waves in basic shear flow, was derived, whose coefficients $S_{0}$ and $R_{00}$ depend on the basic shear flow $U(y)$ and linear topography $h_{b} y$. The variation of topography with latitude $y$ is one of the factors inducing solitary Rossby waves in zonal shear flow, and the shear flow $U(y)$ influences the formation of solitary Rossby wave. 


\section{References}

Benney, D. J., 1966. Long nonlinear waves in fluid flow. Journal of Mathematical Physics 45: 52-63.

Boyd, J. P., 1980. Equatorial solitary waves. Part 1: Rossby solitons. Journal of Phys. Oceanogr. 10: 1 699-1 717.

Clarke, A., 1971. Solitary and cnoidal planetary waves. Geophysical Fluid Dynamics 2: 343-354.

Guo, B. L. and X. F. Pang, 1987. Solitary Waves. Science Press, Beijing. p. 1-363. (in Chinese)

Jeffrey, A. and T. Kawahara, 1982. Asymptotic Methods in Nonlinear Waves Theory. Pitman Publishing Inc, London. p. 256-266.

Larsen, L. N., 1965. Solitary waves in the westerlies. $J$. Atmos. Sci. 22: 222-224.

Li, M. K., Y. J. Hou, B. S. Yin, J. B. Song and W. Zhao, 2006. Numerical simulation of scatterometer assimilated wind and ocean wave in eastern China seas and adjacent waters. Chinese Journal of Oceanology and Limnology 24: 42-47.

Liu, S. K. and B. K. Tan, 1992. Rossby waves with the change of $\beta$. Applied Mathematics and Mechanics 13: 35-44. (in Chinese with English abstract)

Long, R., 1964. Solitary waves in the westerlies. J. Atmos. Sci. 21: 197-200.

Luo, D. H., 1991. Nonlinear Schrödinger equation in the 万r产数新居 barotropic atmosphere and atmospheric blocking. Acta Meteor. Sinica 5: 587-597.

Luo, D. H., 1995. Solitary Rossy waves with the beta parameter and dipole blocking. Journal of Applied Meteorological Science 6: 220-227. (in Chinese with English abstract)
Maslowe, S. A. and L. G. Redekopp, 1980. Long nonlinear waves in stratified shear flows. J. Fluid Mech. 101: 321-348.

Redekopp, L. G, 1977. On the theory of solitary Rossby waves. J. Fluid Mech. 82: 725-745.

Redekopp, L. G and P. D. Weidman, 1978. Solitary Rossby waves in zonal shear flows and interactions. J. Atmos. Sci. 35: 790-804.

Su, J. Z., M. K. Li, Y. J. Hou, B. S. Yin and G. H. Fang, 2006. Surface circulation derived from drifting buoys in midand low-latitude Pacific. Chinese Joumal of Oceanology and Limnology 24: 333-337.

Sun, J., C. L. Guan and J. Shi, 2006. The effect of wave breaking on surface wave imaging by Synthetic Aperture Radar. Chinese Journal of Oceanology and Limnology 24: 21-27.

Wadati, M., 1973. The modified Korteweg-de Vries equation. J. Phys. Soc. Japan 34: 1 289-1 296.

Wang, B. and H. Y. Weng, 1981. Geophysical Fluid Dynamics. China Ocean Press, Beijing, p. 1-531. (in Chinese)

Zhao, Q., 1997. The influence of topography on the ultralong Rossy waves in the tropical atmosphere. J. Trop. Mete. 13: 140-145. (in Chinese with English abstract)

Zhao, Q., Z. T. Fu and S. K. Liu, 2001. Equatorial Envelope Rossby Solitons in a shear flow. Advance in Atmos. Sci. 18: 418-428.

Zhu, B. Z., F. F. Jin and Z. Y. Liu, 1991. An Introduction to the Nonlinear Dynamics of the Atmosphere and Ocean. China Ocean Press, Beijing. p. 1-328. (in Chinese) 